\title{
Regularized, Continuum Yang-Mills Process and Feynman-Kac Functional Integral
}

\author{
M. Asorey^ and P. K. Mitter
}

Laboratoire de Physique Théorique et Hautes Energies`^, Université Pierre et Marie Curie (Paris VI), 4 Place Jussieu, F-75230 Paris Cedex 05, France

\begin{abstract}
Giving an ultraviolet regularization and volume cut off we construct a nuclear Riemannian structure on the Hilbert manifold $\mathfrak{M}$ of gauge orbits. This permits us to define a regularized Laplace-Beltrami operator $\Delta$ on $\mathfrak{M}$ and an associated global diffusion in $\mathfrak{M}$ governed by $\Delta$. This enables us to define, via a Feynman-Kac integral, a Euclidean, continuum regularized Yang-Mills process corresponding to a suitable regularization (of the kinetic term) of the classical Yang-Mills Lagrangian on $T \mathfrak{M}$.
\end{abstract}

\section{Introduction}

In order to make a serious study of non-perturbative quantum Yang-Mills theory in four dimensional space-time, it appears to be indispensable to construct first a regularized Euclidean Yang-Mills theory via a well defined functional integral. This first step has so far only been achieved in the context of lattice gauge theories $[21,11,19]$, no rigorous construction in the continuum with cut-offs having yet been given. The serious block to a direct continuum construction appears to have been the nature of the configuration space of Yang-Mills theory which is the space of gauge orbits [16]. This space is not a linear space and in it we have no globally defined notion of a free field. Thus, as should be obvious, we cannot interpret the cut-off Yang-Mills theory as a free field plus regularised perturbation. There exists a formal quantization procedure going back to de Witt, Feynman, Faddeev-Popov [7] which has enjoyed much success in perturbation theory. But this procedure, in its present form even with cut-offs, is fraught with ambiguities at all levels, measure theoretic and geometric, and cannot be considered as a viable alternative to lattice gauge theories for non-perturbative considerations.

In this paper we shall see how some of these problems can be overcome; in fact we shall give a rigorous definition of a global Euclidean Feynman-Kac integral with regularization working directly in the space of gauge orbits. Our construction

* On leave of absence from Zaragoza University (Spain)

$\star \star$ Laboratoire associé au CNRS 
is directly motivated by the formal Faddeev-Popov quantization procedure and leads to a rigorous continuum alternative to Hamiltonian lattice gauge theory [11]. In [17] it was shown, in an appropriate mathematical set up, that the space of gauge orbits $\mathfrak{M}$ was a smooth Hilbert manifold and in [16] it was shown that the classical Yang-Mills dynamical system is properly defined on $T \mathfrak{M}$, the tangent bundle of the gauge orbit manifold. As such the kinetic term displays a (weak) Riemannian structure on the gauge orbit manifold [16]. In [1] the role of this (weak) Riemannian structure in formal Faddeev-Popov path integral is clearly explained. Once this relationship is understood, we can take our clue from the finite dimensional context (see $[5,2]$ for heuristic considerations) where a rigorous probabilistic set up exists [15]. In that context the aim is to set up Brownian motion (diffusion) in the manifold corresponding to the Riemannian structure i.e. the generator is the Laplace-Beltrami operator [15]. A further potential (suitable globally defined function on the manifold) can then be added via a Feynman-Kac integral. In this paper this approach is generalized to the Hilbert manifold of gauge orbits by introducing suitable volume cut-off and ultraviolet regularization. The volume cut-off is put in by compactifying 3-space as a box with periodic boundary conditions (identified with a 3-torus). The introduction of the ultraviolet regularization is more subtle, so we digress further.

The role of the ultraviolet regularization is two-fold. First with its help we introduce a true $C^{\infty}$ Riemannian structure $\mathbf{g}$ on $\mathfrak{M}$, the Hilbert manifold of gauge orbits of $[17,16]$. This replaces the weak Riemannian structure mentioned earlier. The second role of the ultraviolet cut-off is to permit the introduction of a globally defined $C^{\infty}$ field $\mathbf{G}$ of positive, self adjoint (with respect to $\mathbf{g}$ ), nuclear operators on $\mathfrak{M}$ (the operators acting, for each $[A] \in \mathfrak{M}$, on $T_{[A]} \mathfrak{M}$ ). With its help we obtain globally, Gaussian measures on tangent spaces and Wiener measures on the space of paths in each tangent space. When the ultraviolet cut-off is taken off, $\mathbf{g}$ collapses to the weak Riemannian structure and $\mathbf{G}$ to the identity operator. [The ultraviolet regularization has a Lagrangian interpretation (it is a variant of higher spatial covariant derivative regularisation) and will not violate Osterwalder-Schrader positivity.] The triple $(\mathfrak{M}, \mathbf{g}, \mathbf{G})$ may be called a nuclear Riemannian manifold and such triples appear to be suitable objects for analysis in Hilbert manifolds. In particular we are able to construct rigorously a second order invariant differential operator $\Delta$ on $\mathfrak{M}$ which plays the role of a Laplace-Beltrami operator.

The next step is to construct a strong-Markov process $\mathfrak{X}_{t}$ in $\mathfrak{M}$ (Brownian motion) whose generator is $\Delta$. This is achieved, first locally and then globally by patching up, using Ito stochastic integrals and integral equations in Hilbert space (model space) with smooth "diffusion coefficients" fabricated out of $\mathbf{g}$ and $\mathbf{G}$ in local charts. The global construction parallels the finite dimensional case [15] with minor modifications and a convenient simplification due to the parallelizability of Hilbert manifolds. As a by-product we obtain measures on $\mathfrak{M}$ itself (Remark 4.6). The final step is to define the Euclidean Yang-Mills process via a well defined Feynman-Kac integral employing the potential (magnetic term) which turns out to be a continuous function on $\mathfrak{M}$. This construction resolves the problem posed in [16]. The reconstruction of the quantum theory will be given in the sequel.

Several comments are in order. Our work may be viewed as a concrete model of infinite-dimensional stochastic geometry. It is closely related to [4] which 
served as direct inspiration. Brownian motion in Banach manifolds with abstract Wiener structure has been constructed in [14]. Working in a Hilbert manifold we are able to avoid many of the complications of [14]. The construction of a stochastic process in the space of connections has been discussed in [9] but the point of view of that discussion is not directly related to the Yang-Mills dynamical system. Also Singer has advocated the use of $\zeta$-function regularization to define differential operators on the orbit space (lecture at Cargèse, 1979). Both Singer and, independently, Narasimhan have emphasized the importance of a global approach to continuum gauge field quantization.

\section{Classical Yang-Mills Dynamical System with Space Cut-Off [17, 16]}

A. Let $M_{4}=\mathbb{R} \times V$ be Euclidean space-time with volume cut-off on 3-space $V$. For simplicity we take $V$ as a finite box in $\mathbb{R}^{3}$ with periodic boundary conditions. Thus $V$ can be identified with the 3-torus $S^{1} \times S^{1} \times S^{1}=T^{3}$. Let $P(V, \mathscr{G})$ be a $C^{\infty}$ principal $\mathscr{G}$-bundle on $V$, where $\mathscr{G}$ is a compact, connected simple Lie group. Let $\mathscr{\mathscr { A }}_{k}$ be the $C^{\infty}$ Hilbert manifold of all generic (irreducible) connections in $P$ in Sobolev class $k$, with $k>3$ and even for technical reasons. Let $\tilde{\mathfrak{F}}_{k+1}$ be the Hilbert Lie group of gauge transformations, in Sobolev class $(k+1),[17,16]$. Let $\mathscr{Z}$ be the subgroup of $\mathfrak{G}_{k+1}$ with values in the center of $\mathscr{G}$ and denote $\overline{\mathfrak{G}}_{k+1}=\mathfrak{G}_{k+1} / \mathscr{Z} \cdot \overline{\mathfrak{G}}_{k+1}$ has $C^{\infty}$ free action on $\overline{\mathscr{A}}_{k}$. We have the following

Theorem 1.1. $[17,18,16]$

$$
\pi: \overline{\mathscr{A}}_{k} \rightarrow \overline{\mathscr{A}}_{k} / \overline{\mathfrak{G}}_{k+1} \equiv \mathfrak{M}
$$

with $\pi$ the canonical projection is a $C^{\infty}$ locally trivial principal $\overline{\mathfrak{F}}_{k+1}$ bundle and $\mathfrak{M}$ is a $C^{\infty}$ Hilbert manifold.

A $C^{\infty}$ atlas is given in $[17,16]$. A related theorem was first announced in [20] in the context of $C^{\infty}$ topologies.

It is easy to prove the following:

Proposition 1.2. The manifold $\mathfrak{M}$ is a connected, metrizable, separable topological space with a countable topological base.

In fact a true Riemannian structure for $\mathfrak{M}$ will be constructed in Sect. 2 .

In the principal bundle $\overline{\mathscr{A}}_{k}\left(\mathfrak{M}, \overline{\mathfrak{F}}_{k+1}\right)$ we have a canonical connection, the "Coulomb connection" of [18] (see also [16]), whose horizontal space at a point $A$ is the orthogonal supplement in $T_{A} \overline{\mathscr{A}}_{k}$ of the tangent space $V_{A}$ to the fiber through $A$, [orthogonality with respect to the canonical (weak) invariant $L^{2}$ Riemannian structure on $\left.\overline{\mathscr{A}}_{k}\right]$. It is proved in [18] that this gives a splitting. The restriction of this weak Riemannian structure on the horizontal subspace defines a weak Riemannian structure $g^{w}$ on $\mathfrak{M},[1,16]$.

B. The classical Yang-Mills dynamical system [16] is described by the Lagrangian:

$$
\mathbf{L}=-\frac{1}{2} g_{[A]}^{w}([\dot{A}],[\dot{A}])-\mathscr{V}([A])
$$

which gives a function $T \mathfrak{M} \rightarrow \mathbb{R}$ where

$$
\mathscr{V}([A])=\frac{1}{2}\|F(A)\|_{L^{2}}^{2}
$$


for any $A \in[A]$ and $\|F(A)\|_{L^{2}}$ is the canonical invariant $L^{2}$ norm of the curvature 2-form $F(A)$.

We use throughout the notation $[A]$ to denote an orbit through $A$.

Note that the Lagrangian (1.1) is nothing but the Lagrangian:

$$
\mathbf{L}=-\frac{1}{2}\|\dot{A}\|_{L^{2}}^{2}-\frac{1}{2}\|F(A)\|_{L^{2}}^{2}
$$

which is a function on $T \overline{\mathscr{A}}_{k}$ descended to $T \mathfrak{M}$ using the Coulomb connection. The Gauss condition $d_{A}^{*} \dot{A}=0$ then gets automatically implemented. See [16] for details.

\section{Nuclear Riemannian Structure on $\mathfrak{M}$}

\section{A. Let $k>3$ and even throughout}

Let $\Gamma_{m}$ be the Hilbert space of sections of ad $P \times T^{*} V$ in Sobolev class $m \in \mathbb{Z}$. We can define for $-k+2 \leqq m \leqq k$

$$
\Delta_{A}=d_{A} d_{A}^{*}+d_{A}^{*} d_{A}
$$

as a continuous linear operator: $\Gamma_{m} \rightarrow \Gamma_{m-2}$, for any $A \in \overline{\mathscr{A}}_{k}$. By Sobolev embedding, $\overline{\mathscr{A}}_{k} \subset C^{1}(\overline{\mathscr{A}})$, the space of $C^{1}$ irreducible connections.

Lemma 2.1. For each positive real $\Lambda$ and $-k+2 \leqq m \leqq k$

$$
I+\frac{\Delta_{A}}{\Lambda^{2}}: \Gamma_{m} \rightarrow \Gamma_{m-2}
$$

is a bounded invertible linear operator.

The proof of this lemma is similar to that of proposition (3.3) of [18].

\section{B. Riemannian Structure on $\mathfrak{M}$}

We define a gauge invariant Riemannian metric $\tilde{\mathbf{g}}$ on $\mathscr{A}_{k}$ consistent with its Hilbert space structure as follows:

For any $\tau, \eta \in T_{A} \overline{\mathscr{A}}_{k}$, we define

$$
\tilde{\mathbf{g}}_{A}(\tau, \eta)=\left(\left(I+\frac{\Delta_{A}}{\Lambda^{2}}\right)^{k / 2} \tau,\left(I+\frac{\Delta_{A}}{\Lambda^{2}}\right)^{k / 2} \eta\right)_{L^{2}},
$$

where $(\cdot, \cdot)_{L^{2}}$ is the canonical, invariant (weak) $L^{2}$ scalar product on $T_{A} \overline{\mathscr{A}}_{k}$. Lemma (2.1) implies that the norm engendered by $\tilde{\mathbf{g}}_{A}$ is compatible with the Sobolev $k$-norm in $T_{A} \overline{\mathscr{A}}_{k}$. By construction (2.1) is gauge invariant and it is easy to show that it is $C^{\infty}$. Hence $\tilde{\mathbf{g}}$ defines a true gauge invariant Riemannian structure on $\overline{\mathscr{A}}_{k}$.

The restriction of the Riemannian structure $\tilde{\mathbf{g}}$ on the horizontal subspaces of the tangent spaces to $\overline{\mathscr{A}}_{k}$, given by the Coulomb connection in $\overline{\mathscr{A}}_{k}\left(\mathfrak{M}, \overline{\mathfrak{G}}_{k+1}\right)$, (Sect. 1), defines a true $C^{\infty}$ Riemannian structure $\mathbf{g}$ on $\mathfrak{M}$.

C. Global Covariance Operator Field on $\mathfrak{M}$

Let $i_{k-1}$ be the inclusion map: $\Gamma_{k} \rightarrow \Gamma_{k-2}$. Recall $\operatorname{dim} V=3$ and so $2>\frac{\operatorname{dim} V}{2}$. Hence $i_{k-1}$ is a Hilbert-Schmidt operator. Using the canonical identification of $T_{A} \overline{\mathscr{A}}_{k}$ with 
$\Gamma_{k}$ we define the operator:

$$
\tilde{\mathbf{S}}_{A} \equiv\left(I+\frac{\Delta_{A}}{\Lambda^{2}}\right)^{-1} \circ i_{k-1}: T_{A} \overline{\mathscr{A}}_{k} \rightarrow T_{A} \overline{\mathscr{A}}_{k} .
$$

It is easy to show by using Lemma (2.1) that $\tilde{\mathbf{S}}_{A}$ is a self-adjoint Hilbert-Schmidt operator in $T_{A} \overline{\mathscr{A}}_{k}$ with respect to its Hilbert space structure defined by $\tilde{\mathbf{g}}_{A}$.

Hence we have a covariance operator $\tilde{\mathbf{G}}_{A}$ defined by:

$$
\tilde{\mathbf{G}}_{A} \equiv \tilde{\mathbf{S}}_{A}^{2}: T_{A} \overline{\mathscr{A}}_{k} \rightarrow T_{A} \overline{\mathscr{A}}_{k},
$$

which is a self-adjoint, positive operator of trace class. By the Minlos theorem [10] this covariance defines a Gaussian measure $\tilde{\mathbf{p}}_{A}$ of mean 0 on $T_{A} \overline{\mathscr{A}}_{k}$, equipped with $\tilde{\mathbf{g}}_{A}$ scalar product, countably additive on the least $\sigma$-algebra generated by cylinder sets.

We also define

$$
\tilde{\mathbf{G}}_{t, A} \equiv t \tilde{\mathbf{G}}_{A}
$$

with real $t>0$ a scaled covariance and $\tilde{\mathbf{p}}_{t, A}$ the corresponding Gaussian measure.

By construction the operator fields $\tilde{\mathbf{S}}, \tilde{\mathbf{G}}, \tilde{\mathbf{G}}_{t}$ on $\overline{\mathscr{A}}_{k}$ defined by (2.2), (2.3) are gauge invariant and $C^{\infty}$ (Fréchet). The restriction of $\tilde{\mathbf{S}}_{A}, \tilde{\mathbf{G}}_{A}, \tilde{\mathbf{G}}_{t, A}$ to the horizontal subspace $H_{A} \subset T_{A} \overline{\mathscr{A}}_{k}$ (with respect to the Coulomb connection) composed with the projection on $H_{A}$ with respect to the $\tilde{\mathbf{g}}_{A}$ scalar product defines in $T_{[A]} \mathfrak{M}$ operators $\mathbf{S}_{[A]}, \mathbf{G}_{[A]}, \mathbf{G}_{t,[A]}$ with the same properties (with respect to $\mathbf{g}_{[A]}$ ) as $\tilde{\mathbf{S}}_{A}, \widetilde{\mathbf{G}}_{A}, \tilde{\mathbf{G}}_{t, A}$ (with respect to $\left.\widetilde{\mathbf{g}}_{A}\right)$. The operator fields $\mathbf{S}, \mathbf{G}, \mathbf{G}_{t}$ on $\mathfrak{M}$, so defined, are also $C^{\infty}$. Corresponding to each covariance $\mathbf{G}_{t,[A]}$ we have a $\sigma$-additive Gaussian measure of mean $0, \mathbf{p}_{t,[A]}$ on $T_{[A]} \mathfrak{M}$. The triple $(\mathfrak{M}, \mathbf{g}, \mathbf{G})$ defines a nuclear Riemannian manifold.

\section{Relationship with Ultraviolet Regularization}

Define

$$
T_{[A]}^{(H)} \mathfrak{M} \equiv \mathbf{S}_{[A]} T_{[A]} \mathfrak{M} \subset T_{[A]} \mathfrak{M}
$$

which is a dense subspace of $T_{[A]} \mathfrak{M}$.

Now we introduce a scalar product $\mathbf{h}_{[A]}$ on $T_{[A]}^{(H)} \mathfrak{M}$ as follows. If $\tau, \eta \in T_{[A]}^{(H)} \mathfrak{M}$ define:

$$
\mathbf{h}_{[A]}(\tau, \eta)=\mathbf{g}_{[A]}\left(\mathbf{S}_{[A]}^{-1} \tau, \mathbf{S}_{[A]}^{-1} \eta\right) .
$$

Then our ultraviolet regularized Lagrangian, corresponding to our classical Lagrangian (1.1) is (for $\left.[\dot{A}] \in T_{[A]}^{(H)} \mathfrak{M}\right)$ :

$$
\mathbf{L}_{\mathbf{Y} . \mathbf{M} .}^{\text {Reg. }}=-\frac{1}{2} \mathbf{h}_{[A]}([\dot{\mathbf{A}}],[\dot{\mathbf{A}}])-\mathscr{V}([\mathbf{A}]) .
$$

Note that the ultraviolet regularization is entirely in the kinetic term and the regularising parameter $\Lambda$ enters both through the Riemannian structure $\mathrm{g}$ and the covariance operator field $G$. The potential $\mathscr{V}$ is a non-negative continuous function on $\mathfrak{M}$. In fact $F(A) \in \Gamma_{k-1}\left(\operatorname{ad} P \times \Lambda^{2}(V)\right)$ which enjoys the Banach algebra property since $k>3$ and $\operatorname{dim} V=3$. When $\Lambda \rightarrow \infty$ we recover the classical YangMills Lagrangian (1.1). Note that the regularisation is entirely space-like and hence reflection positivity will be preserved in the Euclidean field theory at the expense 
of full Euclidean covariance in the presence of $\Lambda$. The relation with higher spatial covariant derivative regularisation becomes clear on exploiting, in (2.7), the definition of $\mathbf{h}_{[A]}, \mathbf{g}_{[A]}$, and $\mathbf{S}_{[A]}$, recalling $K>3$ and even, and restricting to subspace of smooth connections. We get a "Hamiltonian Pauli-Villars regularisation" with higher spatial covariant derivatives appearing in the $\dot{A}^{2}$ term which gets absorbed in the definition of the path space measures for Wiener process in tangent spaces.

\section{Diffusion Coefficients in $\mathfrak{M}$ and a Laplace-Beltrami Operator}

In this section we construct certain objects, called "local diffusion coefficients", which will serve in Sect. 4 as inputs in stochastic integral equations via which we will engender a strong Markov process $\mathfrak{X}_{t}$ in $\mathfrak{M}$. We also define in this section a Laplace-Beltrami operator $\Delta$ on $\mathfrak{M}$ which will be seen to coincide, in Sect. 4 , with the generator of $\mathfrak{X}_{t}$. The parallelizability of Hilbert manifolds, exploited in this section, eliminates a possible obstruction to the global construction of $\mathfrak{X}_{t}$ in $\mathfrak{M}$, with unrestricted smooth atlas.

Let $\left(U_{i}, \varphi_{i}\right)$ be a $C^{\infty}$ atlas for $\mathfrak{M}$. For concreteness we take the $C^{\infty}$ atlas of $[17,16]$ and by Proposition (1.2) it is taken to be countable. The image $\varphi\left(U_{i}\right)$ is then defined via a "local background gauge" $[17,16]$. The model space $E$ for $\mathfrak{M}$ is identified, once and for all, with the (closed) horizontal subspace $[17,16]$ $H_{\mathscr{A}} \subset T_{\AA} \overline{\mathscr{A}}_{k}$, at any fixed point $\stackrel{\circ}{A} \overline{\mathscr{A}}_{k}$, since all $H_{A}$ are isomorphic (e.g. by identifying fixed bases). In fact $E$ can be identified with $T_{[\AA]} \mathfrak{M} \simeq H_{\AA}$, with scalar product $\mathbf{g}_{[\AA]}$. The results of this paper are atlas independent.

\section{A. First Diffusion Coefficient}

Take the $C^{\infty}$ Riemannian structure $\mathbf{g}$ on $\mathfrak{M}$ defined in Sect. 2. Let $(U, \varphi)$ a local chart at $[A] \in \mathfrak{M}$, and denote by

$$
\varphi_{*[A]}: T_{[A]}(U) \rightarrow T_{A}(E) \simeq E
$$

the isomorphism of tangent spaces, with $A=\varphi([A])$. Then for any $u, v \in E$ we define the bounded invertible linear operator $\overline{\mathbf{g}}(\varphi A): E \rightarrow E$ by:

$$
\mathbf{g}_{[A]}\left(\varphi_{*[A]}^{-1} u, \varphi_{*[A]}^{-1} v\right) \equiv \mathbf{g}_{\varphi}(A)(u, v) \equiv\left(u, \overline{\mathbf{g}}_{\varphi}(A) v\right) .
$$

Next we note that by Kuiper's theorem [12], $\mathfrak{M}$ is $g$-parallelizable since $\mathfrak{M}$ is a Hilbert manifold. Let $X_{0}$ be a parallelization of the $\mathcal{O}(\mathfrak{M})$ bundle of orthogonal frames:

$$
X_{0}: \mathfrak{M} \times O(E) \rightarrow \mathcal{O}(\mathfrak{M})
$$

$O(E)$ is the group of orthogonal transformations on $E$. [By Kuiper's theorem $O(E)$ is contractible.] Let us denote by

$$
X: \mathfrak{M} \times E \rightarrow T \mathfrak{M}
$$

the trivialization of $T \mathfrak{M}$ induced by $X_{0}$. If $\left\{e_{i}\right\}$ is an orthonormal basis of $E$, for every $[A] \in \mathfrak{M}$ we have that the sequence $\left\{X_{i}([A])\right\}$ of vectors of $T_{[A]} \mathfrak{M}$ defined by

$$
X_{i}([A])=X\left([A], e_{i}\right)
$$


is an orthonormal basis of $T_{[A]} \mathfrak{M}$ with respect to $\mathbf{g}_{[A]}$, i.e.

$$
\mathbf{g}_{[A]}\left(X_{i}([A]), X_{j}([A])\right)=\delta_{i j} .
$$

We define for each $[A] \in \mathfrak{M}$, the isomorphism

by

$$
\tau_{[A]}: T_{[A]} \mathfrak{M} \rightarrow E
$$

$$
\tau_{[A]} X_{i}([A])=e_{i} .
$$

Define the bounded linear invertible operator:

$$
R_{\varphi,[A]}=\tau_{[A]} \varphi_{*[A]}^{-1}: E \rightarrow E .
$$

Then

Hence

$$
\begin{aligned}
\left(R_{\varphi,[A]} u, R_{\varphi,[A]} v\right)_{E} & =\left(\tau_{[A]} \varphi_{*[A]}^{-1} u, \tau_{[A]} \varphi_{*[A]}^{-1} v\right)_{E} \\
& =\mathbf{g}_{[A]}\left(\varphi_{*[A]}^{-1} u, \varphi_{*[A]}^{-1} v\right) \\
& =\left(u, \overline{\mathbf{g}}_{\varphi}(A) v\right)_{E} .
\end{aligned}
$$

$$
\begin{aligned}
\overline{\mathbf{g}}_{\varphi}(A) & =R_{\varphi,[A]}^{*} R_{\varphi,[A]} \\
\overline{\mathbf{g}}_{\varphi}(A)^{-1} & =R_{\varphi,[A]}^{-1} R_{\varphi,[A]}^{*-1} .
\end{aligned}
$$

In other words $R_{\varphi}$ is the expression in local coordinates of parallelization $X_{0}$ (infinite dimensional generalization of a Vierbein field).

We define the first local diffusion coefficient $\xi_{\varphi}$ in $\varphi(U)$

$$
\xi_{\varphi}(A) \equiv \mathbf{S}_{\varphi,[A]} \mathbf{R}_{\varphi,[A]}^{-1},
$$

where

$$
\mathbf{S}_{\varphi,[A]}=\varphi_{*[A]} \mathbf{S}_{[A]} \varphi_{*[A]}^{-1} .
$$

$\mathbf{S}$ being the Hilbert-Schmidt operator field on $\mathfrak{M}$ of Sect. $2 \mathrm{C}$. It is a routine exercise to verify the following

Lemma 3.1. $\xi_{\varphi}: \varphi(U) \rightarrow \mathscr{H} S(E)$ is $C^{\infty}$. $\mathscr{H} S(E)$ is the Hilbert space of HilbertSchmidt operators with the Hilbert-Schmidt norm $\|\cdot\|_{H \cdot S}$.

Corollary 3.2. (Local Lipschitz Continuity). There exists an open set $V \subset U$ such that for any $A, A^{\prime} \in \varphi(V)$

$$
\left\|\xi_{\varphi}(A)-\xi_{\varphi}\left(A^{\prime}\right)\right\|_{H \cdot S} \leqq c\left\|A-A^{\prime}\right\|_{E}
$$

i.e. $\xi_{\varphi}$ is locally lipschitz.

\section{B. Second Diffusion Coefficient}

The second local diffusion coefficient in $\mathfrak{M}$ will be constructed out of the Riemannian connection corresponding to $\mathrm{g}$ and the first local diffusion coefficient $\xi_{\varphi}$ already constructed.

Let $(U, \varphi)$ be a chart at $[A] \in \mathfrak{M}$. From (3.2) we have for fixed $u, v \in E$ the function

$$
\mathbf{g}_{\varphi}(\cdot)(u, v): \varphi(U) \rightarrow \mathbb{R}
$$


and for each $A \in \varphi(U)$, we have the Fréchet derivative:

$$
\begin{aligned}
\mathbf{g}_{\varphi}^{\prime}(A)(u, v): & E \rightarrow \mathbb{R} \\
w & \mapsto \mathbf{g}_{\varphi}^{\prime}(A)(u, v) w
\end{aligned}
$$

as a bounded linear functional.

Define for every $w, u, v \in E$

$$
\mathbf{g}_{\varphi}^{\prime}(A)(w ; u, v) \equiv \mathbf{g}_{\varphi}^{\prime}(A)(u, v) w .
$$

We now define the Christofel functional $\Gamma_{\varphi}(A)(\cdot, \cdot)$ in the local chart $(U, \varphi)$ as a bounded bilinear map:

$$
\begin{gathered}
\Gamma_{\varphi}(A)(\cdot, \cdot): E \times E \rightarrow E \\
\Gamma_{\varphi}(A)(u, v)=\frac{1}{2} \overline{\mathbf{g}}_{\varphi}(A)^{-1}\left\{\mathbf{g}_{\varphi}^{\prime}(A)(u ; v, \cdot)+\mathbf{g}_{\varphi}^{\prime}(A)(v ; \cdot, u)-\mathbf{g}_{\varphi}^{\prime}(A)(\cdot ; u, v)\right\}
\end{gathered}
$$

for every $u, v \in E$ where the terms in the bracket are considered as elements of $E$ by the Riesz theorem.

More explicitly, for $w \in E$,

$$
\left(w, \overline{\mathbf{g}}_{\varphi}(A) \Gamma_{\varphi}(A)(u, v)\right)_{E}=\frac{1}{2}\left\{\mathbf{g}_{\varphi}^{\prime}(A)(u ; v, w)+\mathbf{g}_{\varphi}^{\prime}(A)(v ; w, u)-\mathbf{g}_{\varphi}^{\prime}(A)(w ; u, v)\right\} .
$$

We now define the second local diffusion coefficient $\sigma_{\varphi}(\cdot)$ by:

$$
\sigma_{\varphi}(A)=-\frac{1}{2} \operatorname{tr} \Gamma_{\varphi}(A)\left(\xi_{\varphi} \times \xi_{\varphi}\right)
$$

where $\operatorname{tr} \Gamma_{\varphi}(A)\left(\xi_{\varphi} \times \xi_{\varphi}\right)$ is the unique vector of $E$ such that for any $u \in E$, and any orthonormal basis $\left\{e_{n}\right\}$ of $E$

$$
\left(u, \operatorname{tr} \Gamma_{\varphi}(A)\left(\xi_{\varphi} \times \xi_{\varphi}\right)\right)=\sum_{n=1}^{\infty}\left(u, \Gamma_{\varphi}(A)\left(\xi_{\varphi} e_{n}, \xi_{\varphi} e_{n}\right)\right) .
$$

Each term in the r.h.s. defines a bounded bilinear functional and can be written in the form $\left(\xi_{\varphi} e_{n}, \bar{\Gamma}_{\varphi, u}(A) \xi_{\varphi} e_{n}\right)$ with $\bar{\Gamma}$ bounded linear operator. The series in (3.18) converges and is independent of choice of orthonormal basis since $\xi_{\varphi}$ is HilbertSchmidt.

It is straightforward to check the following:

Lemma 3.3. $\sigma_{\varphi}: \varphi(U) \rightarrow E$ is $C^{\infty}$.

Corollary 3.4.(Local Lipschitz Continuity). There exists an open set $V \subset U$ such that for any $A, A^{\prime} \in \varphi(V)$ :

$$
\left\|\sigma_{\varphi}(A)-\sigma_{\varphi}\left(A^{\prime}\right)\right\|_{E} \leqq c\left\|A-A^{\prime}\right\|_{E}
$$

Lemma 3.5. (Transformation Property of Diffusion Coefficients). Let $(U, \varphi),(V, \psi)$ be two local charts at $[A] \in U \cap V \neq \Phi$. Define:

$$
\theta=\psi \circ \varphi^{-1}: \varphi(U \cap V) \rightarrow \psi(U \cap V)
$$

(transformation of local coordinates). Let $\left\{\xi_{\varphi}, \sigma_{\varphi}\right\},\left\{\xi_{\psi}, \sigma_{\psi}\right\}$ be local diffusion coefficients of Sects. $A, B$ in charts $\varphi, \psi$. 
Then:

$$
\begin{aligned}
& \xi_{\psi}=\theta^{\prime} \xi_{\varphi} \\
& \sigma_{\psi}=\theta^{\prime} \sigma_{\varphi}+\frac{1}{2} \operatorname{tr} \theta^{\prime \prime}\left(\xi_{\varphi} \times \xi_{\varphi}\right),
\end{aligned}
$$

where the first Fréchet derivative $\theta^{\prime}: E \rightarrow E$, bounded linear operator, is the Jacobian of the coordinate change, $\theta^{\prime \prime}: E \times E \rightarrow E$, bounded bilinear map, is the second Fréchet derivative and $\operatorname{tr}$ is defined as in (3.17) and (3.18).

(3.21) is trivial to prove. In fact,

$$
\xi_{\psi}=\mathbf{S}_{\psi} R_{\psi}^{-1}=\theta^{\prime} \mathbf{S}_{\varphi} \theta^{\prime-1} \theta^{\prime} R_{\varphi}=\theta^{\prime} \xi_{\varphi} .
$$

The proof of (3.22) is by a lengthy computation which we omit.

\section{Laplace-Beltrami Operator}

Let $f: \mathfrak{M} \rightarrow \mathbb{R}$, be $C^{2}$ Fréchet.

Let $(U, \varphi)$ be a local chart at $[A] \in \mathfrak{M}$, and $f_{\varphi}$ the function in the local chart. Then $f_{\psi}^{\prime}(A) \in \mathscr{B}(E, \mathbb{R}), f_{\varphi}^{\prime \prime}(A) \in \mathscr{B}(E \times E, \mathbb{R})$.

We define the second order differential operator $\Delta$ by:

$$
\Delta f_{\varphi}(A)=\frac{1}{2} \operatorname{tr} f_{\varphi}^{\prime \prime}(A)\left(\xi_{\varphi}(A) \times \xi_{\varphi}(A)\right)+f_{\varphi}^{\prime}(A)\left(\sigma_{\varphi}(A)\right) .
$$

Identifying $f_{\varphi}^{\prime \prime}(A)$ with a bounded linear operator $E \rightarrow E$ and $f_{\varphi}^{\prime}(A)$ with a vector in $E$, and $\left(f_{\varphi}^{\prime}(A), \Gamma_{\varphi}\left(\mathbf{S}_{\varphi} \times \mathbf{S}_{\varphi}\right)\right)$ as a bounded linear operator,

$$
\begin{aligned}
\Delta f_{\varphi}(A)= & \frac{1}{2} \operatorname{tr}\left(\xi_{\varphi}^{*}(A) f_{\varphi}^{\prime \prime}(A) \xi_{\varphi}(A)\right)+\left(f_{\varphi}^{\prime}(A), \sigma_{\varphi}(A)\right)_{E} \\
= & \frac{1}{2} \operatorname{tr}\left(\overline{\mathbf{g}}_{\varphi}(A)^{-1} \mathbf{S}_{\varphi}^{*}(A) f_{\varphi}^{\prime \prime}(A) \mathbf{S}_{\varphi}(A)\right) \\
& -\frac{1}{2} \operatorname{tr} \overline{\mathbf{g}}_{\varphi}(A)^{-1}\left(f_{\varphi}^{\prime}(A), \Gamma_{\varphi}\left(\mathbf{S}_{\varphi} \times \mathbf{S}_{\varphi}\right)\right)_{E} .
\end{aligned}
$$

$\Delta$ is perfectly well defined, since $\xi_{\varphi}$ is Hilbert-Schmidt, and is independent of the choice of parallelization $X$ (3.3). It is easy to verify using Lemma 3.5. that $\Delta$ is invariantly defined, i.e. it is invariant under coordinate changes. Moreover for finite dimensions and with $\Lambda \rightarrow \infty$ it collapses to the standard Laplace-Beltrami operator for a Riemannian structure g. We shall call it a regularized LaplaceBeltrami operator and show later that it is the generator of a strong Markov process with values in $\mathfrak{M}$, the diffusion process. This Laplace-Beltrami operator is globally and canonically defined from the nuclear Riemannian structure $(\mathfrak{M}, \mathbf{g}, \mathbf{G})$ in $\mathfrak{M}$.

The intrinsic definition of our Laplace-Beltrami operator is as follows. (This resulted from discussions with Narasimhan.)

Let $d: C^{\infty}(\mathfrak{M} \times \mathbb{R}) \rightarrow C^{\infty}\left(T^{*} \mathfrak{M}\right)$ be the exterior differential operator acting on sections of the vector bundle $\mathfrak{M} \times \mathbb{R}$ (M-functions), and $\Delta_{\mathrm{g}}: C^{\infty}\left(T^{*} \mathfrak{M}\right) \rightarrow C^{\infty}(\mathscr{B}(T \mathfrak{M} \times T \mathfrak{M}, \mathbb{R}))$ be the covariant derivative operator of the Levi-Civita connection of $g$ acting on sections of $T^{* \mathfrak{M}}$, where $\mathscr{B}(T \mathfrak{M} \times T \mathfrak{M}$, $\mathbb{R})$ is the bundle of bounded bilinear forms in $T \mathfrak{M}$. Let $\tau_{\mathbf{g}}$ be the canonical mapping of $\mathscr{B}(T \mathfrak{M} \times T \mathfrak{M}, \mathbb{R})$ into $\mathscr{B}(T \mathfrak{M})$, the vector bundle of bounded operators in tangent spaces with the norm topology, and $\alpha_{\mathbf{G}}$ be the map of $\mathscr{B}(T \mathfrak{M})$ into $\mathscr{B}_{N}(T \mathfrak{M})$ (the bundle of positive nuclear operators with the trace norm topology) defined by

$$
\alpha_{\mathbf{G}}(\mathbf{T})=\mathbf{T G}
$$


for any $\mathbf{T} \in \mathscr{B}(T \mathfrak{M})$. Note that $\mathbf{G}$ is a section of $\mathscr{B}_{N}(T \mathfrak{M})$. We define the LaplaceBeltrami operator $\Delta$ of $(\mathfrak{M}, \mathbf{g}, \mathbf{G})$ by

$$
2 \Delta=\operatorname{tr}_{\mathbf{g}} \alpha_{\mathbf{G}} \tau_{\mathbf{g}} \nabla_{\mathbf{g}} d,
$$

where $\operatorname{tr}_{\mathbf{g}}$ is the map of $\mathscr{B}_{N}(T \mathfrak{M})$ in $\mathfrak{M} \times \mathbb{R}$ defined by taking the trace of the operators in $\mathscr{B}_{N}(T \mathfrak{M})$, i.e. the definition of $\Delta$ is such that the following diagram of continuous maps of sections:

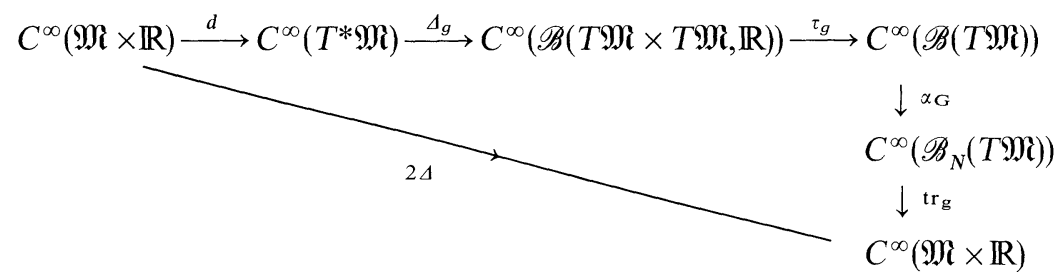

is commutative. It is easy to show that $\Delta$ satisfies (3.23) and (3.24) in local coordinates.

\section{Diffusion Process in $\mathfrak{M}$}

\section{A. Stochastic Integral Equations in Hilbert Space [3]}

In Sect. 2 we gave a family of covariances $\mathbf{G}_{t,[\AA]}$ on $T_{[\AA]} \mathfrak{M} \simeq E$ each $\mathbf{G}_{t,[\AA]}$ being a positive, self-adjoint, nuclear operator with respect to the metric $\mathbf{g}_{[\AA]}$. Hence on the model space $E$ of $\mathfrak{M}$ we have a family of $\sigma$-additive Gaussian measures; parametrized by $t, p_{t}$ of mean 0 and above covariance, i.e. $\left(E, \mathbb{B}(E), p_{t}\right)$ is a probability space where $\mathbb{B}(E)$ is the least $\sigma$-algebra generated by cylinder sets. Let $\Omega_{E}=C^{0}([0, \infty) \rightarrow E)$ be the path space and $\mathbb{B}\left(\Omega_{E}\right)$ the least $\sigma$-algebra generated by cylinder sets. Then $\left(\Omega_{E}, \mathbb{B}\left(\Omega_{E}\right), P\right)$ is Wiener space with $P$ Wiener measure, $P$ being uniquely determined as follows.

Let $\mathbf{W}_{t}: \Omega_{E} \rightarrow E$, measurable, be defined by the evaluation map $\mathbf{W}_{t}(\omega)=\omega(t)$. Then we demand (i) for $t>s, \mathbf{W}_{t}-\mathbf{W}_{s}$ is distributed according to $p_{t-s}$, (ii) for $0=t_{0}$ $<t_{1}<\ldots<t_{k}$ the random variables $\left\{\mathbf{W}_{t_{i}}-\mathbf{W}_{t_{l-1}}\right\}$ are independently distributed. The Wiener process $\mathbf{W}_{t}$ defines $P$ uniquely, via the Kolmogorov construction. Let $\left\{e_{i}\right\}$ an orthonormal basis in $E$ consisting of eigenvectors of $S_{[\AA]}=G_{[\AA]}^{1 / 2}$. Let $P_{n}$ be orthogonal projector of rank $n$ in $E$, defined by $P_{n} u=\sum_{i=1}^{n}\left(e_{i}, u\right) e_{i}, u \in E$. We define a sequence of random variables, $\beta_{t}^{(n)}: \Omega \rightarrow P_{n} E$ by $\beta_{t}^{(n)}(\omega)=\mathbf{S}^{-1} P_{n} \omega(t)$. We take $\beta_{t}^{(n)}$ to be distributed according to law $P$. In particular the components $\beta_{t}^{(n) i}$ are distributed with mean 0 and covariance $\min \left(t_{1}, t_{2}\right) I_{P_{n} E}$. Note that $\mathbf{S} \beta_{t}^{(n)}$ converges to $\mathbf{W}_{t}$ in $\mathbf{L}^{2}(\Omega, d P)$, as $n \rightarrow \infty$.

Let $\mathbb{B}_{s} \subset \mathbb{B}\left(\Omega_{E}\right)$ be the $\sigma$-subalgebra generated by $\mathbf{W}_{t}, t \leqq s$. It is an increasing family (with increasing $s$ ) and $\bigcup_{s} \mathbb{B}_{s}=\mathbb{B}(\Omega)$. We now define non-anticipating operators:

$$
\zeta(\cdot, \cdot):[0, \infty) \times \Omega \rightarrow \mathscr{H} S(E)
$$


jointly measurable in both variables and for each $t$,

$$
\zeta_{t}=\zeta(t, \cdot): \Omega \rightarrow \mathscr{H} S(E)
$$

is $\mathbb{B}_{t}$ measurable.

We define the $\mathbf{L}_{n \cdot a \cdot 0}^{2}([0, t] \times \Omega)$ space of non-anticipating operators by completing in the norm:

$$
\|\zeta\|_{\mathbf{L}_{n \cdot a \cdot o}^{2}}^{2}=\mathscr{E}\left(\int_{0}^{t} d s\left\|\zeta_{S}(\cdot)\right\|_{H \cdot S}^{2}\right)<\infty
$$

where $\mathscr{E}$ is the Wiener expectation.

The Ito stochastic integral of $\zeta,[3]$,

$$
I_{t}(\zeta)=\int_{0}^{t} \zeta_{s}(\cdot) d \beta_{s}(\cdot)
$$

is a bounded linear map into the space of random variables with values in $E$

$$
I_{t}: \mathbf{L}_{n \cdot a \cdot o}^{2}([0, t] \times \Omega) \rightarrow \mathbf{L}^{2}(\Omega, d P),
$$

satisfying

(i) $I_{t}(\zeta)$ non-anticipating (in fact a continuous martingale)

(ii) $\mathscr{E}\left(\left\|I_{t}(\zeta)(\cdot)\right\|_{E}^{2}\right)=\mathscr{E}\left(\int_{0}^{t} d s\left\|\zeta_{s}(\cdot)\right\|_{H \cdot S}^{2}\right)$ (isometry)

(iii) $\mathscr{E}\left(I_{t}\right)=0$

(iv) continuity in $t$, a.s.

(v) $\mathscr{E}\left(\sup _{0 \leqq s \leqq t}\left\|I_{s}(\zeta)(\cdot)\right\| \geqq \lambda\right) \leqq \lambda^{-2} \mathscr{E}\left(\int_{0}^{t} d s\left\|\zeta_{s}(\cdot)\right\|_{H \cdot S}^{2}\right)$.

This $I_{t}(\zeta)(\cdot)$ defines a continuous stochastic process in $E$.

The construction of such a stochastic integral proceeds via finite dimensional approximations. We define $\int_{0}^{t} P_{n} \zeta(s) P_{n} d \beta_{s}^{(n)}$ as standard Ito stochastic integral. Then the limit $n \rightarrow \infty$ exists in $\mathbf{L}^{2}(\Omega, d P)$ and this limit defines the stochastic integral above.

We next state Ito's formula in Hilbert space.

Ito's formula [3].

Let $f: E \rightarrow \mathbb{R}, C^{2}$, and $A \in E$ fixed vector. Consider the stochastic process in $E$

$$
X_{t}=A+\int_{0}^{t} \zeta_{s} d \beta_{s}+\int_{0}^{t} d s \sigma_{s}
$$

where $\zeta$ is a non-anticipating operator with $\|\zeta\|_{\mathbf{L}_{n \cdot a \cdot o}^{2} \cdot \infty}<\infty$ and $\sigma$ a non-anticipating vector in $E$. Then:

$$
\begin{aligned}
f\left(X_{t}\right)= & f(A)+\int_{0}^{t}\left(\zeta_{s}^{*} f^{\prime}\left(X_{s}\right), d \beta_{s}\right)_{E} \\
& +\int_{0}^{t} d s\left(\left\langle f^{\prime}\left(X_{s}\right), \sigma_{s}\right\rangle+\frac{1}{2} \operatorname{tr} f^{\prime \prime}\left(X_{s}\right)\left(\zeta_{s} \times \zeta_{s}\right)\right) .
\end{aligned}
$$

Ito stochastic integral equations in Hilbert space [3]. 
Let

$$
\xi: \mathrm{E} \rightarrow \mathscr{H} S(E)
$$

and

$$
\sigma: E \rightarrow E
$$

be Lipschitz continuous maps. Then we define the stochastic integral equation:

$$
X_{t}=A+\int_{0}^{t} \xi\left(X_{s}\right) d \beta_{s}+\int_{0}^{t} d s \sigma\left(X_{s}\right),
$$

where $X_{t}$ is restricted lie in the space of non-anticipating vectors in $E$.

Theorem 4.1. [3]. There exists a unique solution of (4.5) which defines a continuous strong Markov process in $E$.

First one proves existence and uniqueness for finite dimensional approximations employing projectors $P_{n}$ (defined earlier), by Picard's method, employing 4.2(ii) with convergence in $\mathbf{L}^{2}(\Omega, d P)$. Simple Markov property [6] for the finite dimensional approximations follows from uniqueness and strong Markov property [6] from uniqueness and the Dynkin-Hunt statement that $\beta_{t}^{(n)}$ starts afresh at Markov times. The finite dimensional approximations converge in $\mathbf{L}^{2}(\Omega, d P)$ as $n \rightarrow \infty$ to a unique solution which by construction satisfy the strong Markov property [3].

\section{Transformation of Stochastic Integral Equation}

Let $\theta: E \rightarrow E, C^{2}$. Then

$$
\begin{aligned}
\theta\left(X_{t}\right)= & \theta(A)+\int_{0}^{t} \theta^{\prime}\left(X_{s}\right) \xi\left(X_{s}\right) d \beta_{s} \\
& +\int_{0}^{t} d s\left\{\theta^{\prime}\left(X_{s}\right) \sigma\left(X_{s}\right)+\frac{1}{2} \operatorname{tr} \theta^{\prime \prime}\left(X_{s}\right)\left(\xi\left(X_{s}\right) \times \xi\left(X_{s}\right)\right)\right\} .
\end{aligned}
$$

The transformation law (4.6) follows from Ito's formula (4.4) applied to (4.5) to components of $\theta$ in a basis.

Lemma 4.2. Let $B_{A}=\left\{A^{\prime} \in E,\left\|A^{\prime}-A\right\|_{E}<r\right\}$.

Let $X_{t}$ be the solution of (4.5) [Theorem 4.1].

Let $\tau_{B}=\inf _{t}\left\{t \mid X_{t} \notin B_{A}\right\}$ be the exit time of $X_{t}$ from $B_{A}$.

Then

$$
P\left\{\tau_{B} \leqq \varepsilon\right\}=O\left(\varepsilon^{2}\right)
$$

The proof follows on using (a) Ito's formula (4.4) applied to (4.5) for the function $\|\cdot\|_{E}^{2}: E \rightarrow \mathbb{R}$, (b) property $4.2(\mathrm{v})$, and (c) the Tchebycheff inequality. A similar estimate in the abstract Wiener case is given in [14].

We now turn to the construction of the

\section{B. Local Diffusion Process in $\mathfrak{M}$}

Let $(U, \varphi)$ be a local chart in $\mathfrak{M}$. By virtue of corollary 3.2 and 3.4 there exists a restricted chart $(V, \varphi)$ with $\bar{V} \subset \stackrel{\cup}{U}$ such that the diffusion coefficients $\xi_{\varphi}, \sigma_{\varphi}$ defined 
in Sect. 3 are Lipschitz continuous maps in $\varphi(V)$ having global Lipschitz extensions in $E$.

Let $\tilde{\xi}_{\varphi}, \tilde{\sigma}_{\varphi}$ be any global Lipschitz extensions in $E$ of $\xi_{\varphi}$ and $\sigma_{\varphi}$.

We now take the stochastic integral equation in $E$ :

$$
\tilde{X}_{t}=A+\int_{0}^{t} \tilde{\xi}_{\varphi}\left(\tilde{X}_{s}\right) d \beta_{s}+\int_{0}^{t} \tilde{\sigma}_{\varphi}\left(\tilde{X}_{s}\right) d s
$$

with $A \in \varphi(V)$. By theorem (4.1) the unique solution defines a continuous strong Markov process starting at $A$.

Let $\tau_{V}$ be the Markov exit time from $\varphi(V)$. We denote by $X_{t}$ the process $\tilde{X}_{t}$ considered up to $\tau_{V}$. (For restrictions of processes, see [6], $\xi 1$, Chap. X.)

This process $X_{t}$, the local diffusion process in $\varphi(V)$, is a continuous strong Markov process [6] and is independent of the choice of extension as in the $\mathbb{R}^{n}$ case [15].

We now define [15]:

$$
\mathfrak{X}_{t}^{(V)} \equiv \varphi^{-1}\left(X_{t}\right)
$$

the local diffusion in $V$.

Note that this stochastic process $\mathfrak{X}_{t}^{(V)}$ is defined on the probability space $\left(\Omega_{E}, \mathbb{B}\left(\Omega_{E}\right), P\right)$ with values in $V$.

\section{Global Diffusion Process in $\mathfrak{M}$}

We have the following compatibility of local diffusion processes constructed in Sect. 4B.

Lemma 4.3. Let $\left(V_{1}, \varphi_{1}\right)$ and $\left(V_{2}, \varphi_{2}\right)$ be two local charts at $[A] \in \mathfrak{M}$, where the charts $\left(V_{i}, \varphi_{i}\right)$ are as in Sect. $4 B$. Let $[A] \in W \subset V_{1} \cap V_{2}, W$ an open set. Let $\mathfrak{X}_{t}^{W, 1}, \mathfrak{X}_{t}^{W, 2}$ be the corresponding local diffusions in $W$ starting at $[A]$ obtained via charts $\left(W, \varphi_{1}\right)$, $\left(W, \varphi_{2}\right)$ respectively. Then

$$
\mathfrak{X}_{t}^{W, 1}=\mathfrak{X}_{t}^{W, 2} .
$$

The proof follows from the transformation property (4.6) with $\theta$ being a $C^{\infty}$ extension of $\varphi_{2} \circ \varphi_{1}^{-1}$ to $E$ and the transformation law of local diffusion coefficients given by lemma (3.5).

Note that because of the parallelizability of $\mathfrak{M}$, we were able to choose the first diffusion so as to satisfy (3.21) as a consequence of which the compatible stochastic processes $\mathfrak{X}_{t}^{W, 1}$ and $\mathfrak{X}_{t}^{W, 2}$ are defined using always the same Wiener processes $\beta_{t}^{(n)}$ in $P_{n} E$. We thus avoid the complication of "admissable charts" in Banach manifolds with abstract Wiener structure encountered in [14].

The construction of the global diffusion process in $\mathfrak{M}$ follows the Ito scheme for the finite dimensional case given in six steps in Chap. 4.3 of [15]. Step 1 of [15] has been taken care of in Sect. 4B. Step 2 is standard. Step 3 is taken care of by Lemma 4.3. Note that we always have the same Wiener process $\beta_{t}$ as remarked after Lemma 4.3. For Step 4, the estimate of our Lemma 4.2 replaces the McKean estimate. Step 5 goes through because of our Lemma 4.2 and Lemma III.3 of [14]. Step 6 is standard. The global diffusion process is defined up to a (random) explosion time $\zeta$ as in [15]. 
As a consequence we have the following theorem describing global diffusion in $\mathfrak{M}$ associated to the nuclear riemannian structure $(\mathfrak{M}, \mathbf{g}, \mathbf{G})$

Theorem 4.4. For any $[A] \in \mathfrak{M}$ there exists a unique global continuous strong Markov process $\mathfrak{X}_{t}^{[A]}$ in $\mathfrak{M}$, (i.e. with values in $\left.\mathfrak{M}\right)$, starting at $[A]$, defined on the probability space $\left(\Omega_{E}, \mathbb{B}\left(\Omega_{E}\right), P\right)$ up to a random explosion time $\zeta$, and such that for every local chart $(U, \varphi)$ of $\mathfrak{M}$ and any stopping time $\tau<\zeta$ with $\mathfrak{X}_{\tau} \in U$, the process $X_{t}=\varphi\left(\mathfrak{X}_{t}^{[A]}\right)$ satisfies

$$
X_{t+\tau}=X_{\tau}+\int_{0}^{t} \xi\left(X_{s+\tau}\right) d \beta_{s}+\int_{0}^{t} \sigma\left(X_{s+\tau}\right) d s
$$

with $t+\tau<\tau_{U}$ (the exit time from $U$ ).

\section{Infinitesimal Generator}

Let $\mathbf{B}$ be the Banach space of bounded measurable functions on $\mathfrak{M}$. Then the semigroup $T_{t}$ corresponding to the Markov process $\mathfrak{X}_{t}$ in $\mathfrak{M}$ is defined in the standard fashion on $\mathbf{B}$ :

$$
T_{t} f([A])=\mathscr{E}\left(f\left(\mathfrak{X}_{t}^{[A]}\right)\right),
$$

where $\mathscr{E}$ is the restriction of the standard Wiener expection with respect to $\left(\Omega_{E}, \mathbb{B}\left(\Omega_{E}\right), P\right)$ to the set $\{\omega \mid t<\zeta(\omega)\}$.

Proposition 4.5. The action on bounded $C^{2}$ functions of the infinitesimal generator of the global diffusion $\mathfrak{X}_{t}^{[A]}$ of Theorem 4.4 is given by the Laplace-Beltrami operator (3.24) in any local chart $(U, \varphi)$ at $[A]$ with $\varphi(U)$ a bounded set.

The proof follows from Lemma 4.2 for $\tau_{U}$ which permits localization, Ito's formula (4.4), and (4.2), (ii) and (iii). See Theorem III.2 of [14].

Remark 4.6. The process $\mathfrak{X}_{t}$ of Theorem (4.4) is independent of the choice of parallelization in Sect. 3A, since by Proposition 4.5 its generator is (3.2.4) which is independent of choice of parallelization.

Remark 4.7. As in [4] the global diffusion $\mathfrak{X}_{t}^{[A]}$ in $\mathfrak{M}$ engenders, via its transition functions, a family of probability measures $\mu_{t,[A]}$ on $\mathfrak{M}$.

$$
\left.\mu_{t,[A]}(B) \equiv p(t,[A], B) \equiv P\left(\mathfrak{X}_{t}^{[A]} \in B\right)\right)
$$

for any Borel set $B$ in $\mathfrak{M}$. In general different members of the family $\left\{\mu_{t,[A]}\right\}$ are inequivalent measures on $\mathfrak{M}$.

\section{Regularized Yang-Mills Stochastic Process and Feynman-Kac Integral}

We now define the regularized Yang-Mills process, taking account of the potential $\mathscr{V}$ in (1.1 and 1.2) via a Feynman-Kac integral. We define its semi-group $\mathbf{T}_{t}^{\mathbf{Y} . \mathbf{M} .}$ on $\mathbf{B}$, the Banach space of bounded measurable functions on $\mathfrak{M}$, by:

$$
\mathbf{T}_{t}^{\mathrm{Y} . \mathrm{M}} f([A])=\mathscr{E}\left(f\left(\mathfrak{X}_{t}^{[A]}\right) e^{\left.-\int_{0}^{t} d s \mathscr{V}\left(\mathfrak{X}_{s}^{[} A\right]\right)}\right),
$$

where $\mathscr{E}$ is restricted to the subset $\{t<\zeta\}$ of $\Omega$. 
Note that $\mathscr{V}$ is a continuous, non-negative function on $\mathfrak{M}$ (Sect. 2-D) and hence (5.1) is well defined.

Let $\left\{f_{i} \in \mathbf{B}\right\}, i=1, \ldots, k$, and $0<t_{1}<\ldots<t_{k}<T$. We define the expectation of $\left\{f_{i}\right\}$ at $\left\{t_{i}\right\}$ by:

$$
\begin{gathered}
\left\langle f_{1}\left(\left[A_{t_{1}}\right]\right) \ldots f_{k}\left(\left[A_{t_{k}}\right]\right)\right\rangle_{\Lambda, V} \\
\equiv \lim _{T \rightarrow \infty} Z_{T}^{-1} \mathscr{E}\left(f_{1}\left(\mathfrak{X}_{t_{1}}^{[A]}\right) \ldots f_{k}\left(\mathfrak{X}_{t_{k}}^{[A]}\right) e^{-\int_{0}^{I} d s \mathscr{V}\left(\mathfrak{X}_{s}^{[A]}\right)}\right) \\
Z_{T} \equiv \mathscr{E}\left(e^{-\int_{0}^{T} d S^{\mathscr{V}}\left(\mathfrak{X}_{s}^{[A]}\right)}\right)
\end{gathered}
$$

$\mathscr{E}$ is restricted to the subset $\{T<\zeta\} \subset \Omega$.

In the final formula (5.2) we have emphasized the dependance on cut-offs which must $\rightarrow \infty$ in the end. In particular we cannot hope to have a finite $\Lambda \rightarrow \infty$ limit without $\Lambda$-dependent counter terms to redefine the basic measures. The spacevolume $V$ cut-off was put in right at the start in Chap. I and the ultraviolet regularization in Chap. II. The ultraviolet cut-off in (5.2) appears both through the Wiener measure $P$ on $\Omega_{E}$ as well as the covariance (2.3 and 2.2) which is built into the structure of the diffusion process $\mathfrak{X}$.

Concluding Remark 5.1. In this article we have constructed a diffusion process $\mathfrak{X}_{t}$ in $\mathfrak{M}$ which is adapted to the kinetic term of the regularized Lagrangian (2.8) and via a Feynman-Kac integral we use the rest in order to define a continuum Yang-Mills stochastic process. Since there are no "free gauge fields" in $\mathfrak{M}$ one avenue towards the reconstruction of the quantum theory is the following: Take $\mathbf{L}^{2}\left(d \mu_{t,[A]}, \mathfrak{M}\right)$ were $\mu_{t,[A]}$ is the probability measure on $\mathfrak{M}$ engendered by $\mathfrak{X}_{t}$ (Remark 4.7). Construct a continuous Markov process $\mathfrak{J}_{t}$ whose semigroup is symmetric in $\mathbf{L}^{2}\left(d \mu_{t,[A]}, \mathfrak{M}\right)$ and furthermore leaves $\mu_{t,[A]}$ invariant. $\mathfrak{J}_{t}$ is then automatically stationary. $\mathfrak{I}_{t}$ can be used instead of $\mathfrak{X}_{t}$ to write the Feynman-Kac integral with a compensating change of potential. We shall then be in position to reconstruct the quantum theory via the Osterwalder-Schrader construction. This will be taken up in a sequel to this paper.

Acknowledgements. We have benefitted from conversation with G. F. Dell'Antonio, J. Glimm, L. Gross, S. K. Mitter, M. S. Narasimhan, E. Speer, K. Symanzik, and S. R. S. Varadhan.

\section{References}

1. Babelon, O., Viallet, C.M. : Phys. Lett. 85B, 246 (1979)

2. Cheng, K.S.: J. Math. Phys. (N.Y.) 13, 1723 (1972)

3. Daletskii, Ju.L.: Russ. Math. Surv. 22, 1 (1967)

4. Daletskii, Ju.L., Shnaiderman, Ya.I.: Funct. Anal. Appl. 3, 156 (1969)

5. de Witt, B.: Rev. Mod. Phys. 29, 377 (1957)

6. Dynkin, E.B.: Markov processes. Vols. I and II. Berlin: Springer 1965

7. Faddeev, L.D.: Theor. Math. Phys. (USSR) 1, 3 (1969)

8. Fröhlich, J. : Cargèse lectures. In: Recent Developments in Gauge Theories, Hooft, G.'t et al. (eds.). New York: Plenum Press 1980

9. Gaveau, B., Trauber, P.: C.R. Acad. Sc. Paris 289A, 609 (1979)

10. Gelfand, I.M., Vilenkin, Ya.: Generalized functions. Vol. 4. New York: Academic Press 1964 
11. Kogut, J., Susskind, L.: Phys. Rev. D9, 3501 (1974)

12. Kuiper, N.H.: Topology 3, 19 (1965)

13. Kuo, H.H.: Pac. J. Math. 41, 469 (1972)

14. Kuo, H.H.: Trans. Am. Math. Soc. 169, 439 (1972)

15. McKean, H.P.: Stochastic integrals. New York: Academic Press 1969

16. Mitter, P.K. : Cargèse lectures. In: Recent developments in gauge theories, Hooft, G.t et al. (eds.). New York: Plenum Press 1980

17. Mitter, P.K., Viallet, C.M.: Commun. Math. Phys. (to be published)

18. Narasimhan, M.S., Ramadas, T.R.: Commun. Math. Phys. 67, 21 (1979)

19. Osterwalder, K., Seiler, E.: Ann. Phys. 110, 440 (1978)

20. Singer, I.M.: Commun. Math. Phys. 60, 7 (1978)

21. Wilson, K.: Phys. Rev. D10, 2445 (1974)

Communicated by J. Glimm

Received July 1, 1980 